\title{
Primary Vaginal Calculus in a Woman with Disability: Case Report and Literature Review
}

\author{
Pietro Castellan, MD, Michele Nicolai, MD, Piergustavo De Francesco, MD, \\ Luciano Di Tizio, MD, Roberto Castellucci, MD, Maida Bada, MD, Michele Marchioni, MD, \\ Luca Cindolo, MD, FEBU, and Luigi Schips, $\mathrm{MD}^{3}$
}

\begin{abstract}
Background: Vaginal stones are rare and often unknown entities. Most urologists may never see a case in their careers.

Case Presentation: We present the case of a 34-year-old bedridden Caucasian woman with mental and physical disabilities who presented with a large primary vaginal calculus, which, surprisingly, had remained undiagnosed until the patient suffered a right renal colic caused by a ureteral stone. The vagina was completely filled and a digital examination was not possible. For this reason, the stone was removed using surgical pliers with some maneuvering. A vesicovaginal fistula was excluded, as well as foreign bodies or other nidi of infection. After, urethral lithotripsy was performed as planned. The postoperative course and follow-up were uneventful.

Conclusion: Although vaginal calculi are extremely rare in literature, their differential diagnosis should be considered in women with incontinence and associated disabilities, paraplegia, or prolonged immobilization in recumbent position.
\end{abstract}

Keywords: primary calculus, vaginal calculus, paraplegia, disability, urinary incontinence

\section{Introduction and Background}

V AGINAL CALCULI ARE rarely found and usually misdiagnosed owing to their poor detectability. Depending on the absence or presence of foreign bodies, they are classified as either primary or secondary vaginal stones. The cause of primary vaginal stones is probably to be identified in the pooling and stasis of urine within the vagina, whereas secondary vaginal stones likely result from the deposition of urinary constituents around a nidus. Urethrovaginal fistulas are the most frequent cause and other etiologies are rare, as evidenced by the negligible number of reports identified by a Medline search spanning from 1950 to the present.

We present an extremely rare case of a large primary vaginal calculus resulting from urine statis in a disabled young woman.

\section{Presentation of Case}

A 34-year-old woman hospitalized in the neurology unit was referred to our urology department with fever and a diagnosis of left hydronephrosis. The patient was healthy at birth but during the first months of life suffered seizures typical of West syndrome, a condition that causes severe mental injury, resulting in quadriplegia and complete urinary and fecal incontinence. The patient had experienced frequent urinary tract infections and was noncollaborative. Physical examination revealed soft abdomen and normal externa genitalia. The hymen was intact and a hard brown abnormal mass was observed in the vaginal cavity. Transabdominal sonography revealed normal uterus, cervix, and adnexas, with left kidney grade II hydronephrosis. The bladder and right kidney appeared normal. The abdominal radiograph showed a large $(5 \mathrm{~cm}$ in diameter) radiopaque mass in the vaginal cavity (Fig. 1) and a left distal ureteral stone $(6 \mathrm{~mm}$ in diameter). On account of the patient's condition and immobilization, an abdominal CT scan was not performed. Given the presence of persistent fever and wishing to reduce any risks of serious infection $\left(>38^{\circ} \mathrm{C}\right.$, WBC: $15,0007 \times 10^{3} / \mu \mathrm{L}$, HB: $13 \mathrm{~g} / \mathrm{dL}$, serum creatinine $0.5 \mathrm{mg} / \mathrm{dL}$, other laboratory tests within normal ranges), a left ureteral stent was placed, although endoscopic maneuvering was made somewhat

Departments of ${ }^{1}$ Urology and ${ }^{2}$ Obstetrics and Gynecology, ASL02 Abruzzo, SS. Annunziata Hospital, Chieti, Italy.

${ }^{3}$ Department of Urology, University G.D'Annunzio, Chieti, Italy.

(C) Pietro Castellan et al. 2017; Published by Mary Ann Liebert, Inc. This is an Open Access article distributed under the terms of the Creative Commons Attribution License, which permits unrestricted use, distribution, and reproduction in any medium, provided the original work is properly cited. 


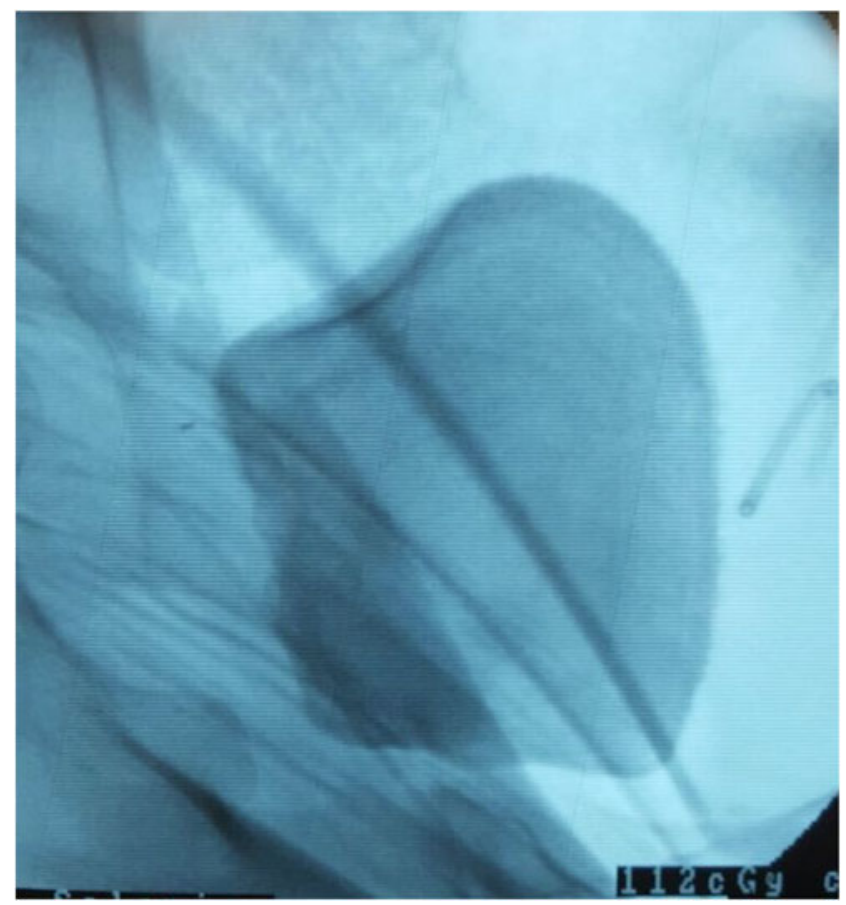

FIG. 1. Rx fluoroscopy imaging of the vaginal calculus during the urethral catheterization.

difficult by the presence of the vaginal stone. For this reason, a month later, the urethral lithotripsy was planned as a second step after removing the vaginal stone. Under general anesthesia, a more detailed examination of the external genitalia was carried out, showing normal vulvar anatomy. The hymen was torn manually to achieve a better access to the vaginal cavity, which appeared entirely filled by a brown, laminated, and parallelepiped-shaped mass with an offensive odor (Fig. 2) that precluded digital examination. With some difficulty, the large, round, and hard vaginal stone,

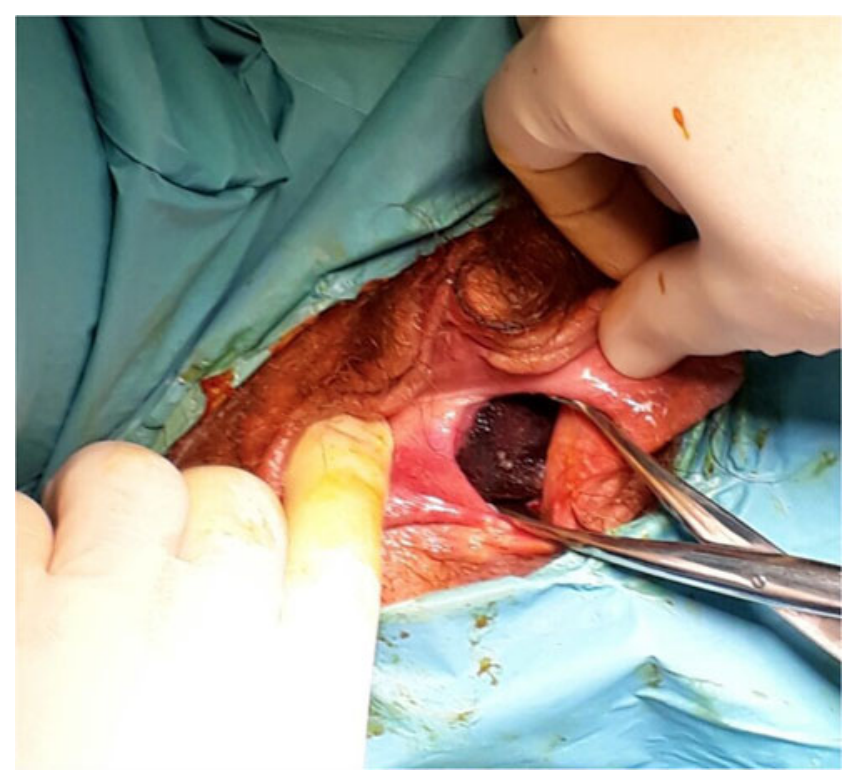

FIG. 2. Vaginal calculus is seen through hymenal orifice in the vagina during its removal. $\sim 5.1 \times 3.7 \times 3.1 \mathrm{~cm}$ and $156 \mathrm{~g}$, was removed using surgical pliers and several maneuvers (Fig. 3). No episiotomy was performed during the procedure, and after removing the stone, a small laceration of the vaginal roof was sutured. The presence of fistulas between the bladder and vagina was investigated and excluded by cystoscopy and urethrocystography. After this first step, a left ureteroscopy was finally performed, confirming the presence of a 6-mm stone in the ureteral pelvic tract, which was removed by laser lithotripsy. A left Double-J ureteral stent was left in place with external strings. On chemical-physical analysis, the calculus appeared to be composed of $100 \%$ struvite (ammonium magnesium phosphate). The left ureteral stent was accidently removed on the first day after surgery, otherwise the postoperative period was uneventful and the patient was discharged 3 days later.

\section{Discussion and Literature Review}

The first articles concerning primary vaginal stones date back to the 1950s. Since then, research has been geared toward classifying the disease and establishing its etiology.

In the literature, vaginal stones are more frequently associated with urogenital tract abnormalities such as vesicovaginal or urethrovaginal fistulas, urethrovesical dysfunction, ectopic vaginal ureter, or vaginal obstructions/strictures. They are usually more frequent in children or young women because, under normal conditions, the anatomy and physiology of the vagina do not allow the formation of stone. Vaginal calculi can be divided into primary and secondary calculi. Primary calculi are caused by chronic presence of urine inside the vagina that leads to the deposition of urinary components and aggregation of salt crystals. Other causes may be neuropathic bladder, bladder exstrophy, myelodysplasia, cerebral palsy, or mental retardation associated with urinary incontinence and urine stasis. Again, vaginal outlet obstructions, such as imperforate hymen, ectopic ureter, urethral diverticulum, or hypospadias, as well as occlusions developed after surgery or as a result of menopausal atrophy

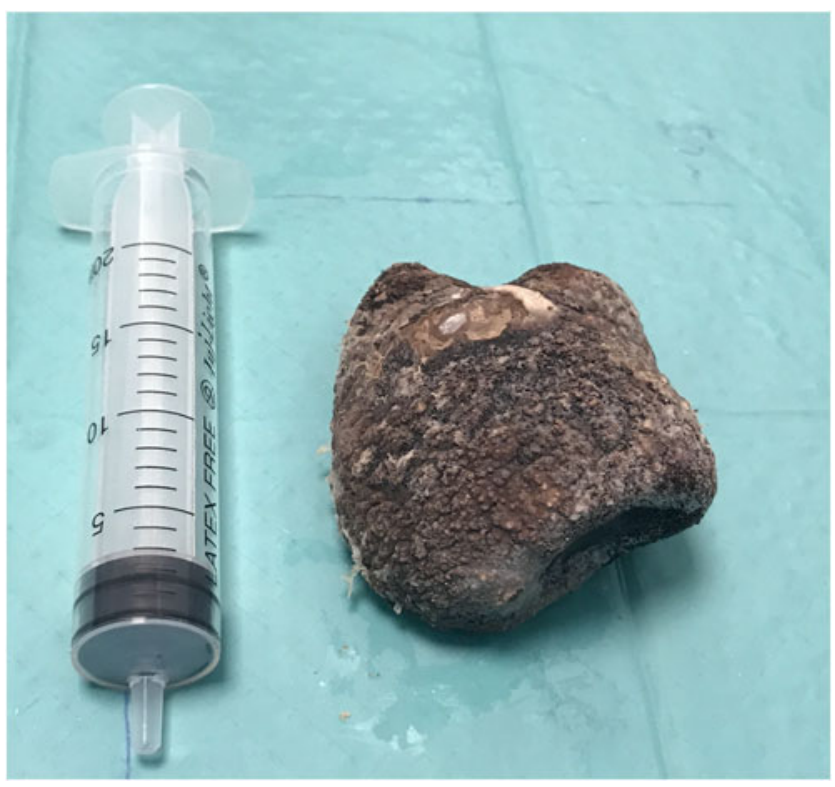

FIG. 3. The large round hard vaginal stone $(\sim 5.1 \times$ $3.7 \times 3.1 \mathrm{~cm})$ removed from the vagina. 
Table 1. Review of Literature Reporting Vaginal Primary Stone in Woman with Disabilities

\begin{tabular}{|c|c|c|c|c|c|}
\hline Author & Year & $\begin{array}{l}\text { Patient } \\
\text { age }\end{array}$ & Comorbidity & $\begin{array}{l}\text { Misdiagnosed } \\
(y \text { or } n)\end{array}$ & Initial wrong diagnosis \\
\hline Avsar et al. ${ }^{1}$ & 2013 & 22 years & Paraplegia & $\mathrm{Y}$ & $\begin{array}{l}\text { Laparoscopy for an } \\
\text { adnexal mass }\end{array}$ \\
\hline Ikeda et al. ${ }^{2}$ & 2013 & 42 years & $\begin{array}{l}\text { Mental and physical } \\
\text { disabilities }\end{array}$ & $\mathrm{Y}$ & Bladder calculus \\
\hline Jaspers et al. ${ }^{3}$ & 2010 & 5 years & Spastic tetraplegia & $\mathrm{Y}$ & Bladder calculus \\
\hline Cetinkurşun et $\mathrm{al}_{5}{ }_{5}$ & 2001 & 13 years & Cerebral palsy & $\mathrm{Y}$ & Bladder calculus \\
\hline Yoshimura et al..$^{5}$ & 2000 & 11 years & $\begin{array}{l}\text { Mental and physical } \\
\text { disabilities }\end{array}$ & Y & Urinary tract infections \\
\hline
\end{tabular}

$\mathrm{N}=$ no; $\mathrm{Y}=$ yes.

or radiotherapy, could cause primary stone formation. Secondary calculi are caused by the presence of foreign bodies in the vagina including forgotten objects, such as pessaries or intrauterine devices, medical gauzes, or surgical tools. Another likely cause is migration of vesical stones associated with vesicovaginal septum ulceration or with deposition of hemosiderin crystals and desquamated cells around a nidus. The majority of vaginal stone cases have been reported in association with gynecologic conditions (Table 1) and most were misdiagnosed for bladder calculi and planned for open or endourologic treatment. In our case, quadriplegia and complete urinary and fecal incontinence were undoubtedly the underlying causes of stone formation, the latter owing to vaginal pooling associated with a prolonged recumbent position (no fistulas). Vaginal contamination, in turn, predisposes to infections from urease-producing bacteria. In this scenario, bacteria such as Escherichia coli, Proteus mirabilis, and Klebsiella species are responsible for turning a normal, acidic milieu into an alkaline environment, facilitating the precipitation of triple phosphate (struvite) and formation of primary vaginal calculi. In most cases, this production leads to a solitary stone, but multiple stones are also encountered. The growth process is generally slow and size depends on the time it takes for the vaginal cavity to be fully filled. Vaginal stones usually do not cause any specific symptoms or signs, except abdominal or pelvic pain, and thus may go undetected for a long time. Indeed, most calculi are discovered incidentally, during radiological investigations for other conditions and are frequently misdiagnosed as bladder stones.

The importance of differential diagnosis should, therefore, be emphasized in patients with mental-physical disabilities and urinary infections who present with pelvic pain. Such diagnosis should be based on a thorough assessment, including physical examination, ultrasonography, cystoscopy, CT, or magnetic resonance imaging. Since vaginal stones are fairly rare occurrences, most urogynecologists do not even suspect their presence; thus, delayed diagnosis allows more time for growth and makes surgical resolution all the more likely.

In our case, the stone was discovered incidentally by ultrasound examination for a suspected renal colic. Mental and physical disabilities prevented the patient from expressing any complaints, and the urinary tract was examined only on account of persistent fever. Vaginal stones are difficult to detect with ultrasonography alone (i.e., without the assistance of other imaging investigations), but in our case, fluoroscopy images had been obtained earlier at the time of ureteral catheterization. Treatment of vaginal stones involves complete removal, either through manual extraction or by surgical correction. Some authors report a transabdominal or transperitoneal approach with bladder incisions or opening the vaginal anterior wall to preserve the integrity of the genital organs and hymen. However, we opted for vaginal extraction of the entire mass through hymenal incision. Other authors also suggest ultrasonic lithotripsy or intracorporeal shock wave lithotripsy. Before surgical correction, estrogen therapy is recommended to strengthen the epithelium of the vagina and make it more resistant to iatrogenic stretching. In our case, the difficulty in removing the stone caused a small laceration of the vaginal roof, whereas the vagina, bladder, and rectum were undamaged. Late complications may include recurrent infections, urinary incontinence, or urolithiasis. Finally, identifying the underlying factors that led to the development of vaginal stones is essential both to ensure early treatment and to prevent recurrences. Data from urine chemistry and stone analysis provide invaluable information in this respect.

Our report first aims to remind that vaginal stones, although rare, should always be suspected in heavily disabled women with urinary symptoms.

\section{Conclusion}

Vaginal stones are rare entities and most physicians may never see a case throughout their careers. Differential diagnosis should be considered when urinary stones are discovered in women with mental and physical disabilities, particularly those who are bedridden and have urinary incontinence.

\section{Disclosure Statement}

No competing financial interests exist.

\section{References}

1. Avsar AF, Keskin HL, Catma T, et al. A large primary vaginal calculus in a woman with paraplegia. J Low Genit Tract Dis 2013;17:61-65.

2. Ikeda Y, Oda K, Matsuzawa N, Shimizu K. Primary vaginal calculus in a middle-aged woman with mental and physical disabilities. Int Urogynecol J 2013;24:1229-1231.

3. Jaspers JW, Kuppens SM, van Zundert AA, de Wildt MJ. Vaginal stone in a 5-year-old girl: A novel approach to removal. J Pediatr Adolesc Gynecol 2010;23:e23-e25.

4. Cetinkurşun S, Surer I, Demirbag S, Oztürk H. A primary vaginal stone in a disabled child. Obstet Gynecol 2001;98: 978-979. 
5. Yoshimura T, Nagata Y, Matsuura K, Okamura H. Primary vaginal stone in a 11-year-old recumbent girl. Gynecol Obstet Invest 2000;50:64-66.

Address correspondence to:

Pietro Castellan, MD

Department of Urology

ASL02 Abruzzo

SS. Annunziata Hospital

Via dei Vestini

Chieti 66100

Italy

E-mail: castellanpietro@gmail.com
Cite this article as: Castellan $\mathrm{P}$, Nicolai $\mathrm{M}$, De Francesco P, Di Tizio L, Castellucci R, Bada M, Marchioni M, Cindolo L, Schips L (2017) Primary vaginal calculus in a woman with disability: case report and literature review, Journal of Endourology Case Reports 3:1, 182-185, DOI: 10.1089/cren.2017.0100.

\section{Abbreviations Used \\ $\mathrm{CT}=$ computed tomography \\ $\mathrm{HB}=$ hemoglobin \\ $\mathrm{WBC}=$ white blood cells}

